Yusmania Emili Coello

\title{
Estudio fenomenológico de la resiliencia: una visión desde la experiencia del docente universitario
}

\author{
Phenomenological study of resilience: a vision from the \\ experience of the university teacher \\ Yusmania Emili Coello \\ lafisica2009@hotmail.com \\ Universidad Nacional Experimental Francisco de Miranda. \\ Venezuela
}

Recibido: $10 / 07 / 2017$

Aprobado: 25/08/2017

\begin{abstract}
RESUMEN
A continuación se ofrece el estudio de la resiliencia desde la perspectiva del docente universitario. El propósito giró en torno a comprender los significados de resiliencia derivados de la experiencia docente al afrontar situaciones adversas en su vida cotidiana. Se trata de un estudio de naturaleza cualitativa que se desarrolló desde la tradición de la fenomenología hermenéutica de Heidegger (2010). Los resultados indican que la resiliencia es un concepto positivo que equivale a capacidad, habilidad, destreza y competencia humana, que se vale del optimismo, de la perseverancia, de la lucha, del aprendizaje y re-aprendizaje, del vivir y revivir mediante la dinámica de mecanismos emocionales, cognitivos y socioculturales. De allí que, las estrategias empleadas por el docente ante estas situaciones son la automotivación, autocontrol, confianza en sí mismo y en el otro, respecto, humildad, tolerancia; pero principalmente, se maneja con dos elementos clave y complementarios como los son: i) el pensar fenomenológico, que es reflexionar para entender y comprender la situación vivida y poder tomar las decisiones acertadas y favorables y ii) el diálogo abierto y flexible, basado en el preguntar y repreguntar para mediar posiciones, ideas, pensamientos divergentes, valores, entre otros aspectos requeridos al momento de afrontar una realidad adversa.
\end{abstract}

Palabras clave: resiliencia, fenomenología hermenéutica, experiencia docente 


\begin{abstract}
The following is a study of resilience from the perspective of university teachers. The purpose was to understand the meanings of resilience derived from the teaching experience in facing adverse situations in their daily lives. This is a qualitative study that developed from the tradition of Heidegger's (2010) hermeneutic phenomenology. The results indicate that resilience is a positive concept that equals human ability, ability, skill and competence, which is based on optimism, perseverance, struggle, learning and relearning, living and reviving through dynamics Of emotional, cognitive and sociocultural mechanisms. Hence, the strategies used by the teacher in these situations are selfmotivation, self-control, self-confidence and respect, humility, tolerance; But mainly, it is managed with two key and complementary elements as they are: i) phenomenological thinking, which is to reflect to understand and understand the situation and to make the right and favorable decisions; and ii) open and flexible dialogue based on The questioning and re-examination to mediate positions, ideas, divergent thoughts, values, among other aspects required when confronting an adverse reality.
\end{abstract}

Key words: resilience, hermeneutic phenomenology, teaching experience

\title{
INTRODUCCIÓN
}

Hay que reconocer que la resiliencia es una capacidad humana asociada a la entereza más allá de la resistencia. Esto significa que es la habilidad, destreza o competencia adquirida por el individuo que le permite sobreponerse a un estímulo adverso, con actitud y firmeza, con optimismo, y confianza. De allí el hecho de asumir que la resiliencia constituye un proceso dinámico, constructivo, interactivo, sociocultural que se manifiesta en distintos niveles de naturaleza biológica, neurofisiológica y social en atención a estímulos externos.

Se parte del hecho que en la vida hay momentos difíciles, críticos, cargados de crisis, desesperanzas, en los que sentimos que todo está en contra, que estas situaciones llegan de manera imprevista y obligan a tomar decisiones para la cual hay que saber manejar emociones, conocimientos y aspectos socioculturales que inciden en la situación que se afronta. Este manejar es una capacidad que desarrolla el ser humano, pues todo ser es capaz de recuperarse, salir adelante y fortalecido. Es una capacidad 


\section{CIENCIAMATRIA \\ Revista Interdisciplinaria de Humanidades, Educación, Ciencia y Tecnología \\ Año V. Vol. V. №9. Julio - Diciembre 2019 \\ Hecho el depósito de ley: pp201602FA4721 \\ ISSN-L: 2542-3029; ISSN: 2610-802X \\ Universidad Nacional Experimental Francisco de Miranda (UNEFM). Santa Ana de Coro. Venezuela}

Yusmania Emili Coello

humana de superación, bien sea por coraje, talento, espíritu de mejora, entre otras que todos las personas llevamos dentro, Kateb. (2011).

Dicho esto, precisa declarar que el estudio que aquí se presenta trata sobre la resiliencia, cuyo propósito se orienta a comprender los significados de resiliencia derivados de la experiencia docente al afrontar situaciones adversas en su vida cotidiana. Se trata de un estudio de corte cualitativo que se inscribe en la tradición fenomenológica de Martín Heidegger (2010), por tratarse de una metodología con tendencia naturalista e interpretativa orientada a descubrir y comprender los comportamientos y sentidos de la fenómeno; es un proceso que se crea, es un arte, su producto o hallazgos es una obra de arte. Esto es lo que Denzin y Lincoln (2012.p.49) refieren como "investigación bricoleur".

La selección de la fenomenología hermenéutica obedece a que es un método que permite develar lo que está oculto, sacar lo escondido como verdad. Es una tarea investigativa que implica desistir de la apariencia para develar el ser a nivel de su estructura y significatividad; así como, identificar el sentido arrojado en las palabras más profundas manifestadas por el ser, para finalmente, desmontar algo que ya está montado e ir a los elementos fundamentales que lo constituyen.

Por otra parte, hay que destacar que la selección de los informantes clave del estudio requiere de la habilidad del investigador para identificar al sujeto que le proporcione información pertinente sobre el foco que se explora. En este sentido, para el presente estudio se ha decido ubicar como unidad de análisis a tres (03) docentes universitarios correspondientes a la Universidad Politécnica Territorial de Falcón "Alonso Gamero".

\section{DESCRIPCIÓN DE LA SITUACIÓN PROBLEMÁTICA}

La resiliencia es la capacidad que posee el ser humano para afrontar la adversidad; se caracteriza por salir fortalecido, alcanzando un estado de excelencia profesional y personal a partir de la situación vivida. Desde la psicología positiva Seligman (1999), se 


\section{CIENCIAMATRIA \\ Revista Interdisciplinaria de Humanidades, Educación, Ciencia y Tecnología \\ Año V. Vol. V. №9. Julio - Diciembre 2019 \\ Hecho el depósito de ley: pp201602FA4721 \\ ISSN-L: 2542-3029; ISSN: 2610-802X \\ Universidad Nacional Experimental Francisco de Miranda (UNEFM). Santa Ana de Coro. Venezuela}

\section{Yusmania Emili Coello}

considera que las personas más resilientes son aquellas que logran un mayor equilibrio emocional frente a las situaciones de estrés, gestionando mejor la presión. Esto implica autocontrol frente a los acontecimientos y mayor capacidad para afrontar los desafíos.

De lo dicho puede interpretarse que la resiliencia, es el convencimiento que tiene la persona en poder superar los obstáculos de manera exitosa, no imagina la posibilidad de la derrota aunque los resultados estén en contra; a partir de la adversidad surge un comportamiento ejemplar orientado a destacar en situaciones de incertidumbre con resultados altamente positivos.

Se está consciente de que la sociedad actual es el reflejo de que se encuentra dominado hoy en día por la tecnología, la complejidad y la globalización. Ante esta realidad, Grinberg (1999) sostiene su idea en que la necesidad de la figura de un nuevo líder educativo que posea; por un lado, entendimiento y conocimiento; por el otro, desarrolle la habilidad para crear espacios y prácticas en un marco de respeto promoviendo los valores de una nueva sociedad, tales como la democracia, la equidad, la diversidad y la justicia social.

De hecho, el referido autor, al ubicarse en el plano del ser humano, es partidario de una perspectiva de desarrollo co-participativa, en la cual los hombres y mujeres son cocreadores del universo futuro, lo que constituye un reto que el hombre deben asumir con compromiso y responsabilidad, en consecuencia se contempla la evolución armónica del ser humano en las dimensiones de: ser, estar, saber y tener (Grinberg, 2006).

Desde esta perspectiva humana y co-creadora del universo futuro, se inscribe el docente universitario como agente formador y co-creador de conocimientos, conductas, valores y actitudes en los estudiantes. Por consiguiente, conviene pensar la resiliencia como una capacidad innata en este agente, de allí que Valles (2014) considere necesario incluir en la formación del docente aspectos relacionados con la resiliencia. 


\section{CIENCIAMATRIA \\ Revista Interdisciplinaria de Humanidades, Educación, Ciencia y Tecnología \\ Año V. Vol. V. №9. Julio - Diciembre 2019 \\ Hecho el depósito de ley: pp201602FA4721 \\ ISSN-L: 2542-3029; ISSN: 2610-802X \\ Universidad Nacional Experimental Francisco de Miranda (UNEFM). Santa Ana de Coro. Venezuela}

\section{Yusmania Emili Coello}

Esta inclusión es necesaria ante el mundo complejo que se vive hoy en día, en el que se exige mayor capacidad para enfrentar las adversidades, los desafíos del entorno cambiante e inestable y sobre todo, ante la necesidad de hacer uso de la creatividad en situaciones de crisis y conflictos. No obstante, se observa que muchos docentes se dejan invadir por sus emociones ante estas situaciones, demostrando falta de autocontrol para manejarse en escenarios caóticos, razón por la cual Valles (2014) sugiere incluir una formación resiliente que permita la adaptación de la persona resiliente que enfrenta la adversidad, desarrollar una conciencia colectiva humanitaria y la espiritualidad como condición fundamental del docente que promueve experiencias resilientes.

Cabe destacar que, el docente universitario está sometido continuamente a eventos inciertos, indeterminados, conflictivos, caóticos, estresantes y problemáticos dentro de su entorno académico, generando desequilibrios o ruptura del orden emocional manifestado en un comportamiento agresivo, de mal genio, intolerancia, con una actitud negativa, pesimista, encerrados en los errores y defectos más que en las cualidades, indeciso al momento de tomar decisiones, entre otras. Eventos que afectan significativamente el desarrollo de sus relaciones intra e interpersonales, en su convivir diario con colegas y estudiantes, poniendo en riesgo su desempeño personal y profesional dentro de la organización.

Por consiguiente, (Barrientos y colaboradores, 2019, p. 181) plantean que "es importante realizar evaluaciones y consultoría psicológica periódicamente a los docentes para diagnosticar a tiempo una actitud desfavorable, o bien, prevenir sentimientos de malestar y desmotivación que en el futuro ya no se puedan modificar o manejar". Es allí donde las universidades deben generar planes de prevención en la salud integral de los docentes para conocer el alcance de afecciones que puedan estar padeciendo, ante lo cual, podrían generarse estrategias que permitan contribuir en promover una aptitud resiliente en los docentes con la finalidad de articular acciones 
que permitan superar episodios adversos en la vida profesional del docente universitario.

Entendiendo que, la resiliencia es asumida como la capacidad de los seres humanos de surgir a partir del caos y del desorden, dos ingredientes característicos de las situaciones de adversidad. Quiñones (2007.p.19). Dicho en forma sencilla, manejar y gestionar el caos requiere desarrollar la capacidad resiliente en las personas; capacidad que se vincula con el auto-control mental, la auto-conciencia y la agilidad mental que le permita actuar, aprovechar y gestionar la complejidad, el caos y la incertidumbre en su quehacer individual y social.

La resiliencia constituye un área de especial interés, por lo tanto esta investigación parte del supuesto de concebir la condición humana del docente, como protagonista de la actividad educativa universitaria; interesa, entonces, conocer a partir de las vivencias y experiencias propias del docente ¿Cómo afronta y supera situaciones de adversidad?; vale decir, ¿Cómo el docente, a partir de sus propias experiencias personales supera la adversidad?

Cabe destacar que las preguntas de investigación constituyen un componente esencial dentro de los estudios de racionalidad cualitativa; porque guían, orientan el rumbo inicial del esfuerzo de indagación científica, Maxwell (1996). Por otra parte, conviene definir la intencionalidad de esta investigación, para ello precisa declarar como propósito general comprender los significados de resiliencia en relación al contexto universitario, derivados de la experiencia docente al afrontar situaciones adversas en su vida cotidiana.

\section{REFERENCIAL TEÓRICO}

\section{La resiliencia como un proceso de naturaleza humana}

Desde diversas disciplinas como la biología, psicología, entre otras definen la resiliencia como la capacidad del ser humano para hacer frente a las adversidades, superarlas e 


\section{CIENCIAMATRIA \\ Revista Interdisciplinaria de Humanidades, Educación, Ciencia y Tecnología \\ Año V. Vol. V. №9. Julio - Diciembre 2019 \\ Hecho el depósito de ley: pp201602FA4721 \\ ISSN-L: 2542-3029; ISSN: 2610-802X \\ Universidad Nacional Experimental Francisco de Miranda (UNEFM). Santa Ana de Coro. Venezuela}

Yusmania Emili Coello

inclusive salir fortalecidos (Grotberg y Suárez citados en Rosales, 2008, p. 21). Tradicionalmente, es un concepto tomado de la física y está referido a la capacidad de un material de retomar su forma original luego de ser sometido a altas presiones. También es utilizado en medicina, específicamente en el área de osteología, que constituye la propiedad que tiene los huesos de crecer en la orientación correcta posterior a sufrir una fractura (Melillo y Suárez, 2004).

Refiere Valles (2014) que es a partir de la década de los 70, cuando las Ciencias Humanas acogen el término confiriéndole la significación que se maneja actualmente propia de un novel paradigma emergente que se vincula al Modelo de Desafío dejando en el pasado el Modelo del Daño; en este último modelo se analizaban los efectos negativos, producto de heridas y traumas. Mientras que, el Modelo del Desafío convierte lo adverso en desafío para seguir viviendo, creciendo, creando desde una perspectiva holística. Al comparar los dos modelos, se concluye que la resiliencia como fenómeno se ajusta al enfoque del Desafío porque analiza y enriquece la calidad de todos los recursos que contribuyen a restaurar creativamente las experiencias y eventos traumáticos (Rosales, 2008).

En este sentido, Puerta de Klinkert (2002), citado por Valles (2014), expresa acerca de la resiliencia:

Los seres humanos no nos encontramos totalmente desprotegidos y vulnerables ante la fuerza de un evento que en sí mismo puede implicar daño o riesgo: Poseemos una especie de escudo que es la resiliencia...sirve como filtro que atenúa los posibles efectos desagradables o nocivos, y muchas veces logra incluso transformarlos en factores de superación (p.16).

En el recorrido conceptual acerca de la resiliencia como manifestación de la naturaleza humana, se define la resiliencia como la capacidad que tienen las personas para desarrollarse desde una perspectiva psicológica dentro de los límites normales, aun cuando subsisten en entornos de pobreza, riesgo o familias disfuncionales, eventos de 


\section{CIENCIAMATRIA \\ Revista Interdisciplinaria de Humanidades, Educación, Ciencia y Tecnología \\ Año V. Vol. V. №9. Julio - Diciembre 2019 \\ Hecho el depósito de ley: pp201602FA4721 \\ ISSN-L: 2542-3029; ISSN: 2610-802X \\ Universidad Nacional Experimental Francisco de Miranda (UNEFM). Santa Ana de Coro. Venezuela}

Yusmania Emili Coello

estrés prolongado, centros de confinamiento, entre otros ámbitos, (Uriarte, 2005). De acuerdo a esta afirmación, la resiliencia implica superar los perjuicios que acompañan las situaciones de adversidad, contextos desfavorecidos y limitados socioculturalmente. Por su parte, Vanistaendel (2002), define la resiliencia como una cualidad humana universal que está presente en toda persona y situaciones difíciles que permiten hacer frente a las adversidades y salir fortalecido de las experiencias negativas. De allí el considerar que la resiliencia es un conjunto de procesos de naturaleza social e intrapsíquicos que permite la vivencia de una existencia sana en un contexto insano (Rutter, 1993).

Con respecto al planteamiento del párrafo anterior, otros autores coinciden en sus puntos de vista al considerar la aptitud resiliente como el resultado de una conjunción de factores ambientales y el temperamento, asociado a un tipo de habilidad cognitiva que poseen algunas personas aun cuando tiene poca experiencia en los retos que enfrentan (Crespo, 2002).

Por su parte, refiere Valles (2014) que Wolin y Wolin (1993), hacen referencia al concepto de Mandala de la resiliencia y proponen algunas características para las personas resilientes, entre las que destacan las siguientes: introspección, independencia, capacidad de relacionarse, iniciativa, humor, creatividad y moralidad.

En Venezuela, Sambrano (2010), citado por Valles (2014) hace referencia a que la resiliencia tiene dos componentes relevantes: i) la resistencia a la devastación y ii) la capacidad para reconstruir luego de vivir experiencias adversas o encontrarse bajo circunstancias destructivas (p.75). Se tratan de elementos que permiten la construcción de niveles psicológicos para alcanzar una mejor salud mental y enfrentar el porvenir.

Aunado a ello, Sambrano (2010) fundamenta las competencias de la resiliencia a partir de los postulados de la Inteligencia Emocional (Goleman, 2012). Esto obedece a que la inteligencia emocional está referida a la habilidad de conocer e interpretar los sentimientos propios y ajenos, con la finalidad de asumirlos como factores que orientan 
Yusmania Emili Coello

el pensamiento y la acción, principalmente con las capacidades de relacionarse con otros (Interpersonales) y automotivación respectivamente.

\section{La resiliencia como comportamiento asertivo}

La resiliencia se asume como la capacidad emocional, cognitiva y socio cultural que poseen las personas que permite reconocer, enfrentar y transformar las situaciones adversas, problemáticas, inciertas; hecho que permite asumir que la resiliencia constituye un enfoque alternativo que brinda la posibilidad de enfocarse en las fortalezas de las personas. Por otro lado, hay que reconocer que la naturaleza de la resiliencia es compleja; la misma presenta múltiples dimensiones y factores de orden individual, familiar y socio cultural.

La resiliencia como comportamiento asertivo ha sido considerada desde la psicología social, si examinamos los postulados de Goleman (2012), nos damos cuenta que también aborda dentro de sus estudios las emociones y reacciones del individuo ante momentos o situaciones críticas, problemáticas; sin embargo, se asumió a Goleman para entender la inteligencia emocional.

La psicología positiva es considerada como el estudio científico de las fortalezas y virtudes que poseen las personas, que hacen posible adoptar o asumir una perspectiva más abierta en relación al potencial humano, sus motivaciones y capacidades, Seligma (1999). Esto conduce a entender que la vida buena nos lleva al fluir, al desarrollo y a la evolución del ser humano como ente bio-psico-socio-cultural, lo cual implica autoconocimiento de los puntos fuertes, fortalezas del individuo para luego reconfigurar su vida y lograr aplicar sus capacidades de forma asertiva a cada situación que vive.

Lo que importa ahora destacar es la necesidad de desarrollar las fortalezas individuales en las personas con el fin de lograr el fluir (flow) con la vida; un fluir que permita al ser humano hacer frente a la adversidad, a las situaciones problemáticas que vive en su cotidianidad; esto es, ser un individuo resiliente. De igual manera, es necesario comprender las competencias de una persona resiliente con el fin de desarrollar las 


\section{CIENCIAMATRIA \\ Revista Interdisciplinaria de Humanidades, Educación, Ciencia y Tecnología \\ Año V. Vol. V. №9. Julio - Diciembre 2019 \\ Hecho el depósito de ley: pp201602FA4721 \\ ISSN-L: 2542-3029; ISSN: 2610-802X \\ Universidad Nacional Experimental Francisco de Miranda (UNEFM). Santa Ana de Coro. Venezuela}

Yusmania Emili Coello

capacidades que hacen posible superar las adversidades. Entre estas competencias, Pittaluga (2013) destaca seis tipos: inteligencia emocional, práctica, social, autoeficacia, disciplina personal y modelos mentales flexibles.

Otro punto interesante de los planteamientos de Pittaluga (2013) es el pensamiento creativo e innovador como atributos propios de las personas resilientes. Es preciso aclarar que el pensamiento creativo es la expresión del pensar divergente; es producto de la curiosidad donde emerge un flujo de ideas originales y adaptables a la necesidad de resolver enigmas o inquietudes. Es un fluir de muchas posibilidades, múltiples opciones y diversas asociaciones entre las ideas con el fin de combinar y generar una invención e innovación que busca satisfacer alguna necesidad.

\section{HALLAZGOS SIGNIFICATIVOS DE LA RESILIENCIA}

La tarea interpretativa consistió en transcribir los datos, diseñar matrices para identificar, construir y destruir significados que expresen las relaciones y la unidad de sentido en función de los hallazgos sobre la resiliencia como tema de investigación. Para garantizar la confidencialidad de la información y el anonimato del informante, fue conveniente asignar un código a cada uno de ellos. Se procedió a realizar una interpretación de las manifestaciones de los informantes clave para identificar sus categorías y finalmente, realizar la integración de significados de los tres actores en un todo coherente.

Para los informantes clave el ser resiliente funda sus acciones en los valores; por lo tanto, las estrategias de reflexión que emplea en la resolución de problemas se hace en función de diferentes significados a saber: en primer lugar, la reflexión es actividad puramente mental y el tema se relaciona con la filosofía; en segundo lugar, se trata de un acto empírico que se da ciencias tales como la física.

Crear la unidad de sentido en relación al tema de resiliencia; parte de la síntesis interpretativa en función de los hallazgos obtenidos donde los rasgos emocionales se 
CIENCIAMATRIA

Revista Interdisciplinaria de Humanidades, Educación, Ciencia y Tecnología

Año V. Vol. V. №9. Julio - Diciembre 2019

Hecho el depósito de ley: pp201602FA4721

ISSN-L: 2542-3029; ISSN: 2610-802X

Universidad Nacional Experimental Francisco de Miranda (UNEFM). Santa Ana de Coro. Venezuela

Yusmania Emili Coello

presentan en tres planos de acción: en lo personal, social e institucional; pues constituyen los ámbitos de acción del profesor universitario, tal como se muestra en la siguiente matriz:

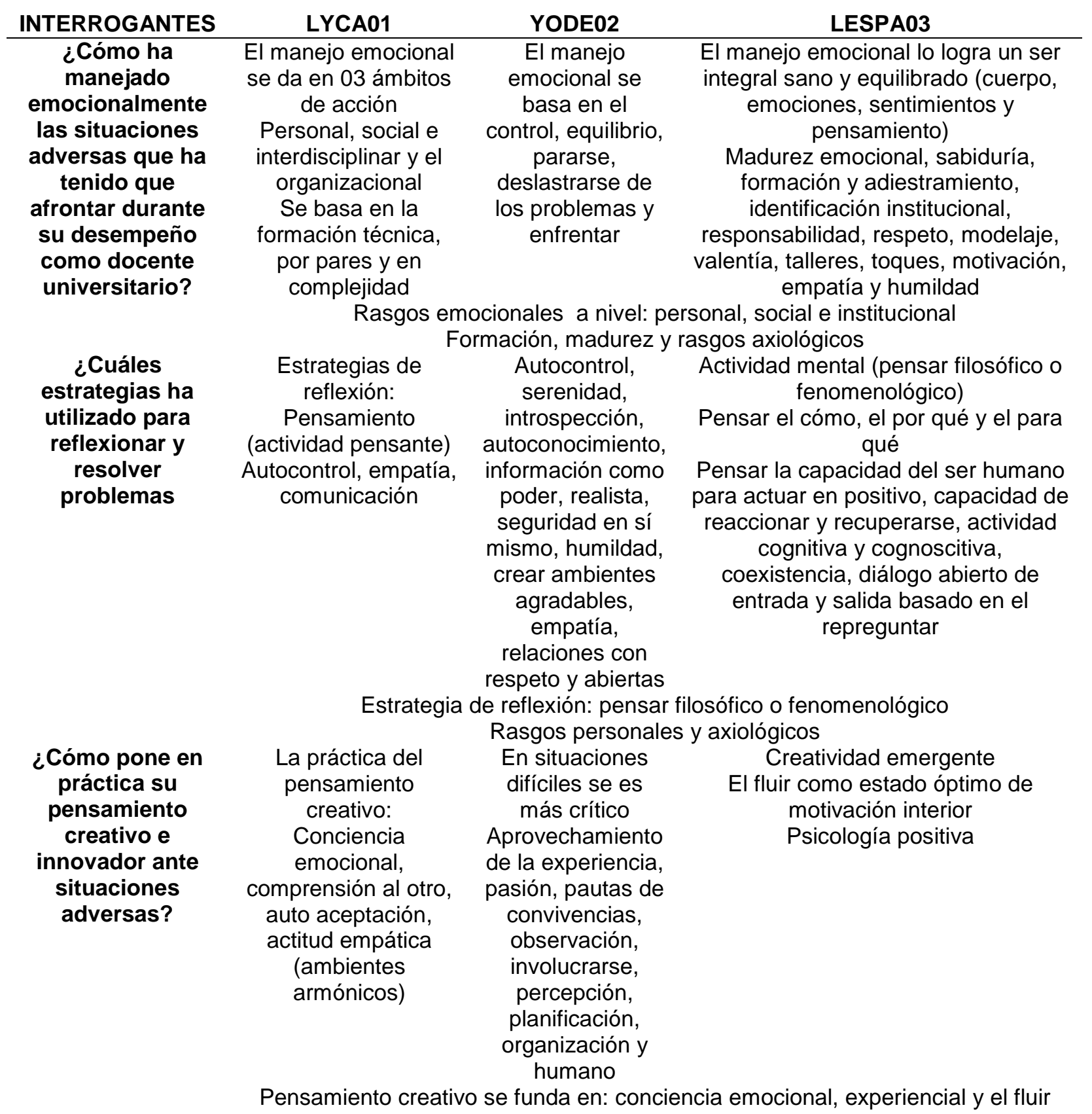




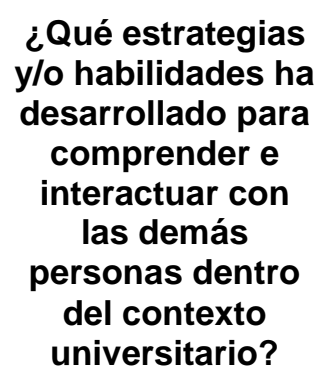

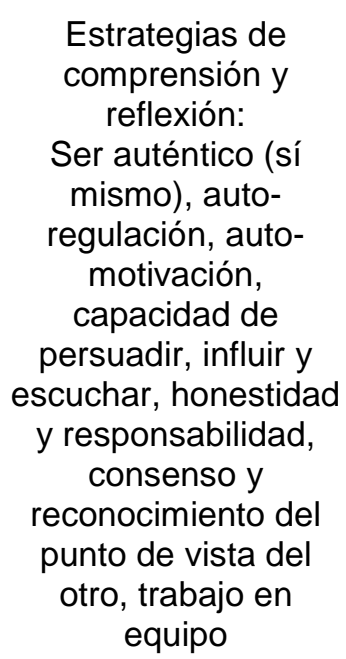

\section{motivacional del YO interior}

Planificación, Reconociendo que las relaciones son observación, información,

formación, conocimiento, compañerismo, solidaridad, respeto autocontrol, cónsono con la que se dice y se hace impredecibles, inciertas e incontrolables

Basado en valores como la dignidad y la humildad

\section{Matriz 1}

Representación visual de los significados de resiliencia

Ahora bien, el ser resiliente se basa en el pensar filosófico o fenomenológico, ya que este pensar considera el asunto con atención y detenimiento para estudiarlo, comprender bien su significado y reflexionar sobre cómo podemos mejorar en lo personal y a las demás personas, a través de la resolución de problemas. El pensar fenomenológico implica no conformarse solo con el cómo y el porqué de las cosas, sino que debe responder a la interrogante reflexiva del el para qué de las cosas porque encierra profundidad.

En este sentido, reflexionar sobre situaciones adversas invita a pensar la relevancia de la capacidad del ser humano para actuar en positivo; por consiguiente, el pensar fenomenológico desarrolla la capacidad para reaccionar y recuperarse ante situaciones ligadas a la adversidad, saliendo reforzados, para afrontar con creatividad y optimismo la vida (actitud positiva). Es por ello que, la solución de las situaciones adversas implica cualidades y habilidades de pensamiento, no hay solución única; todo depende de la naturaleza del problema, circunstancia, ontología. 


\section{CIENCIAMATRIA \\ Revista Interdisciplinaria de Humanidades, Educación, Ciencia y Tecnología \\ Año V. Vol. V. №9. Julio - Diciembre 2019 \\ Hecho el depósito de ley: pp201602FA4721 \\ ISSN-L: 2542-3029; ISSN: 2610-802X \\ Universidad Nacional Experimental Francisco de Miranda (UNEFM). Santa Ana de Coro. Venezuela}

\section{Yusmania Emili Coello}

Desde esta perspectiva, las emociones dentro de la dimensión personal, conducen al ser humano a desarrollar la capacidad de procesar información, de sentir, actuar con sentido y propósito. Es un darnos cuenta de nosotros mismos, de nuestra esencia y existencia y luego del entorno donde operamos y de la experiencia en él; es conciencia pura que invita a anticiparnos, imaginar el futuro y a entender las intenciones de otros Pittaluga (2013). Al respecto Goleman (2012) refiere que el cerebro social posee una infinidad de circuitos concebidos para adaptarse a la mente de otras personas e interactuar con ellas.

Esta dimensión personal incluye el manejo de las emociones a partir de la autoconciencia, entendida esta como el conocimiento y comprensión de nuestra emociones, sentimientos, intenciones, valoraciones y comportamientos, Marchena (2007: 23). Así mismo, el autocontrol constituye una de las capacidades clave para manejar las emociones propias, lo que permite elegir la manera adecuada de expresarlas.

Igualmente, la automotivación influye significativamente en el manejo de las emociones del docente; según Marchena (2007), está asociada al impulso interior, la tenacidad, el disfrute por lo que se hace, la confianza en sí mismo, el optimismo y la perseverancia que fungen como motores dinamizadores para impulsar el éxito en cualquier actividad y sobre todo ante una situación adversa.

Cabe destacar que la dimensión personal da cabida a la social, la cual está asociada a las relaciones interpersonales (Goleman, 2012), vale decir, el interactuar con los otros en los diversos escenarios del contexto universitario en este caso en particular que aquí se estudia. Es establecer relaciones de compenetración o rapport, se trata de un estado que se caracteriza, según Goleman (2012:77) por: i) la atención absoluta y recíproca de las personas que interactúan, ii) la sincronía no verbal y iii) la positividad, que se establece también por el grado de empatía. De allí que, la empatía y la capacidad social 
tienen que ver con "la facultad de gestionar las relaciones con los demás" Goleman (2012:48).

La estrategia más idónea para reflexionar y solucionar problemas es el pensar filosófico o fenomenológico, es un pensar comprensivo. Conviene reseñar, que pensar es imaginar, reflexionar, acto o proceso de tener ideas o pensamientos, incluyendo razonamiento, la solución de problemas y la toma de decisiones. Aquí se asume que pensar es un conjunto de operaciones mentales en cualquier combinación, que incluyen la atención, la imaginación, la percepción, comprensión. Por consiguiente, el pensamiento es una habilidad que se fortalece y se desarrolla con la capacitación sistemática; por ende, influye significativamente en la capacidad del ser humano para hacer frente a las adversidades, vale decir, ayuda a formar seres resilientes.

Entre los valores de la resiliencia, Kateb (2011:89) destaca que "La autoestima es uno de los pilares de la resiliencia", pues las personas que creen en sí mismas tienen una fe irrefutable en sus capacidades, por lo tanto tienen más probabilidades de salir delante de manera más exitosa. Es un valor que parte por incrementar el sentimiento de orgullo de tal forma que aumente también los niveles de autoestima y exigencia ante situaciones difíciles que les toque enfrentar. Se desarrolla mediante una actitud positiva, con un enfoque de optimismo y espiritualidad, viendo las cosas íntegramente en sus verdaderas y exactas relaciones, pensando en cómo resolver sin dejarnos vencer.

En relación al pensamiento creativo se pudo conocer que el mismo se funda en: conciencia emocional, experiencial y en el fluir motivacional del yo interior, lo que implica que este pensar exige un flujo constante de inspiración para elevar la creatividad a partir de las propias fortalezas del ser humano. De la creatividad e innovación se deriva el mejoramiento, en términos de calidad, productividad y en la generación de nuevas ideas adaptable y aplicables en un contexto y/o situación determinada. 


\section{CIENCIAMATRIA \\ Revista Interdisciplinaria de Humanidades, Educación, Ciencia y Tecnología \\ Año V. Vol. V. №9. Julio - Diciembre 2019 \\ Hecho el depósito de ley: pp201602FA4721 \\ ISSN-L: 2542-3029; ISSN: 2610-802X \\ Universidad Nacional Experimental Francisco de Miranda (UNEFM). Santa Ana de Coro. Venezuela}

Yusmania Emili Coello

\section{CONSIDERACIONES FINALES}

Las reflexiones que aquí se ofrecen están relacionadas con resiliencia desde la experiencia del docente universitario. En este sentido, la resiliencia es la capacidad emocional, cognitiva y axiológica que manifiesta el docente ante situaciones difíciles, crisis e inciertas; son capacidades asociadas a la automotivación, autoconciencia, autodominio o autocontrol; así como, a la seguridad, confianza en sí mismo y en el otro. La resiliencia está entrecruzada con el manejo de las emociones, esto indica que al enfrentar situaciones adversas el docente debe aplicar el autocontrol de sus sentimientos, emociones, pasiones y sentires, todo ello con el fin de comprender la situación y tomar las decisiones favorables para salir exitoso del momento difícil que afronta.

Al explorar las experiencias resilientes que posee el docente universitario, se pudo contactar que éste ha vivido situaciones conflictivas con estudiantes, donde ha tenido que poner a prueba su capacidad resiliente. Clasifica el docente sus experiencias en tres (03) grandes ámbitos de acción: personal, social e institucional, los cuales se entrecruzan e interconecta.

La resiliencia es una capacidad del ser humano que se desarrolla mediante la formación y capacitación en materia de los rasgos personales y profesionales, con el fin de mostrar un mejor desempeño en su quehacer educativo. Capacidades que están orientadas a desarrollar habilidades para el manejo emocional, cognitivo y práctico que se requiere en las relaciones interpresonales propias del contexto universitario. Implica el uso de la conciencia, motivación del yo interior, de la creatividad e innovación para hacer frente a los momentos críticos que les toca vivir.

Desde esta perspectiva, entender el significado de la resiliencia, desde la perspectiva del docente, es entender que la resiliencia es un concepto positivo que equivale a capacidad, habilidad, destreza y competencia humana, que se vale del optimismo, de la 


\section{CIENCIAMATRIA \\ Revista Interdisciplinaria de Humanidades, Educación, Ciencia y Tecnología \\ Año V. Vol. V. No9. Julio - Diciembre 2019 \\ Hecho el depósito de ley: pp201602FA4721 \\ ISSN-L: 2542-3029; ISSN: 2610-802X \\ Universidad Nacional Experimental Francisco de Miranda (UNEFM). Santa Ana de Coro. Venezuela}

Yusmania Emili Coello

perseverancia, de la lucha, del aprendizaje y re-aprendizaje, del vivir y revivir mediante la dinámica de mecanismos emocionales, cognitivos y socioculturales.

Finalmente, al intentar comprender cómo afronta y supera situaciones de adversidad el docente, a partir de sus propias experiencias personales supera la adversidad, se concluye que es mediante la automotivación, el autocontrol, la confianza en sí mismo y en el otro, el respecto, la humildad, la tolerancia; pero principalmente, se maneja estratégicamente con dos elementos clave y complementarios como los son: i) el pensar fenomenológico, es reflexionar para entender y comprender la situación vivida y poder tomar las decisiones acertadas y favorables y ii) el diálogo abierto y flexible, para mediar posiciones, ideas, pensamientos divergentes, valores, entre otros aspectos requeridos al momento de afrontar una realidad adversa.

\section{REFERENCIAS CONSULTADAS}

1. Barrientos Oradini, N., Araya Castillo, L., Veloso Salazar, V., \& Herrera Ciudad, C. (2019). Optimismo disposicional en docentes de universidades privadas en región metropolitana en Chile. Revista Arbitrada Interdisciplinaria Koinonía, 4(7), 168-184.

Recuperado

de http://fundacionkoinonia.com.ve/ojs/index.php/revistakoinonia/article/view/199

2. Crespo, C. (2002). La promoción de resiliencia con niños y adolescentes. Entre la vulnerabilidad y la exclusión. Bogotá: Edit. Ecoe.

3. Creswell, J. W. (1998). Diseños de investigación y búsqueda cualitativa. Eligiendo entre cinco tradiciones. Thousand Oaks, USA. Material traducido para fines didácticos por Lic. Indira Paz (Coro, Estado Falcón).

4. Goleman, D. (2012). (2012). El cerebro y la Inteligencia emocional: Nuevos descubrimientos. Madrid. Ediciones B.S.A.

5. Grinberg, J. (1999). El rol del docente en la escuela del nuevo milenio. Adaptación para Eduforum en el Primer Coloquio Universidad Torcuato Di Tella - Universidad de New México. New México, USA. Realizado en octubre 1999. p. 1. 


\section{CIENCIAMATRIA \\ Revista Interdisciplinaria de Humanidades, Educación, Ciencia y Tecnología \\ Año V. Vol. V. No9. Julio - Diciembre 2019 \\ Hecho el depósito de ley: pp201602FA4721 \\ ISSN-L: 2542-3029; ISSN: 2610-802X \\ Universidad Nacional Experimental Francisco de Miranda (UNEFM). Santa Ana de Coro. Venezuela}

Yusmania Emili Coello

6. Grinberg M. (2006). Resignificando el Desarrollo. La Sociedad Inmolada. Boletín Unid@s. Extraído de: www.unida.org.ar/boletín/_boletín18/index.htm. [Consulta: 2012, septiembre 09].

7. Heidegger. M. (2010). Ser y tiempo. México. Fondo de Cultura Económica.

8. Kateb.(2011). Cómo vencer la adversidad. Resiliencia. La fuerza vital para continuar tu camino. España. Editorial Robin Book.

9. Marchena, E. (2007). Gerencie el protocol con inteligencia emocional. Venezuela. Ediciones Z.

10. Maxwell, J. (1996). Diseño de Investigación Cualitativa. Una Aproximación Interactiva. 1era Edición. USA. Sage Publicaciones. Material traducido para fines didácticos por: Lic. Indira Paz.

11. Melillo, A. y Suárez, O. (2004). Resiliencia. Descubriendo las propias fortalezas (1ra. Edición). Buenos Aires: Paidós.

12. Pittaluga, C. (2013). Liderazgo resiliente. La innovación en entornos adversos. Veneuzlea. editorial Galac.

13. Quiñones. M. (2007). Resiliencia. Resignificación creativa de la adversidad. Colombia. Universidad Distrital Francisco José de Caldas.

14. Rosales, G. (2008). Niñez-Resiliencia-Creatividad. Argentina: Edit. Brujas

15. Rutter, M. (1993). Resilience: Some conceptual considerations. Journal of Adolescent Health, 14(8), pp. 626-631.

16. Sambrano, J. (2010). Resiliencia. Transformación positiva de la realidad. Caracas: Edit. Alfa

17. Seligman.M. (1999). The presidents address. APA.1998. Annual Report. American Psychologist, 54, 559-562.

18. Uriarte, J. (2005). Construir la resiliencia en la escuela. Revista de Psicodidáctica, vol. 11. Núm. 1, 2006.pp.7-23. Universidad del País Vasco. España

19. Valles, Y. (2014). Construyendo la resiliencia en el contexto universitario: Una aproximación hermenéutica desde la experiencia del docente. Trabajo de 


\section{CIENCIAMATRIA}

Revista Interdisciplinaria de Humanidades, Educación, Ciencia y Tecnología

Año V. Vol. V. №9. Julio - Diciembre 2019

Hecho el depósito de ley: pp201602FA4721

ISSN-L: 2542-3029; ISSN: 2610-802X

Universidad Nacional Experimental Francisco de Miranda (UNEFM). Santa Ana de Coro. Venezuela

Yusmania Emili Coello

investigación para optar a la categoría de titular. Universidad Politécnica Territorial de Falcón "Alonso Gamero". Coro - Venezuela.

20. Vanistaendel, S.; Lecomte, J. (2002). La felicidad es posible. Barcelona: Gedisa.

21. Wolin, S.J.; Wolin, S. (1993). The Resilient Self: How Survivors of Troubled Families Rise above Adversity. New York: Villard.

(C2019 por el autor. Este artículo es de acceso abierto y distribuido según los términos y condiciones de la licencia Creative Commons Atribución-NoComercial-Compartirlgual 4.0 Internacional (CC BY-NC-SA 4.0) (https://creativecommons.org/licenses/by-nc-sa/4.0/). 